\title{
Clinical Distribution Characteristics of I439 Carbapenem-Resistant Escherichia coli Strains in China: Drug Resistance, Geographical Distribution, Antibiotic MIC50/90
}

Wei Zhang, ${ }^{1,2}$ Zhirong Li, (D) $^{3}$ Na Wang, (ID' Zhicong Yang,' Jia Li,' Caiqing $\mathrm{Li}$, 'Xuying Han, 'Jinlu Liu,' Liping $\mathrm{Li}^{4}{ }^{4}$ Shuwang Wang, ${ }^{4}$ Minghua Zhan ${ }^{1,5}$

'Microbiology Department, The First Affiliated Hospital of Hebei North University, Zhangjiakou, Hebei Province, People's Republic of China; ${ }^{2}$ Clinical Laboratory, State Key Laboratory of Complex Severe and Rare Diseases, Peking Union Medical College Hospital, Chinese Academy of Medical Science and Peking Union Medical College, Beijing, People's Republic of China; ${ }^{3}$ Clinical Laboratory, Hebei Provincial Center for Clinical Laboratories, The Second Hospital of Hebei Medical University, Shijiazhuang, People's Republic of China; ${ }^{4}$ Clinical Laboratory, Zhangjiakou Xuan Gang Hospital, Zhangjiakou, Hebei Province, People's Republic of China; ${ }^{5}$ Clinical Laboratory, Peking University People's Hospital, Beijing, People's Republic of China

Video abstract

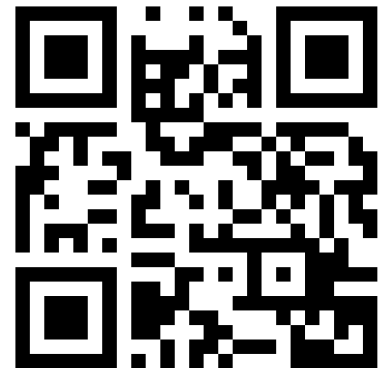

Point your SmartPhone at the code above. If you have a $Q R$ code reader the video abstract will appear. Or use: https://youtu.be/2iknZTw|Xp8

Correspondence: Minghua Zhan

Department of Clinical Laboratory, The First Affiliated Hospital of Hebei North University and Peking University People's Hospital, No. II South Street, $X$ Zhi Men, Beijing, 100044, People's Republic of China

Tel $+86-15531311750$

Fax +86-1088326317

Email minghuazhan02@163.com
Purpose: To explore the clinical distribution characteristics and antimicrobial susceptibilities of carbapenem-resistant Escherichia coli $(C R-E C O)$ in Hebei Province, China, from 2017 to 2019 , and provide data on the treatment of this bacterial infection and the prevention of its spread.

Materials and Methods: A total of 1439 CR-ECO strains were collected from 2017 to 2019 in Hebei Province, China. Drug sensitivity tests were performed using the minimum inhibitory concentration (MIC) method, and the data were analyzed statistically using WHONET5.6 software.

Results: A total of 54,377 strains of Escherichia coli were isolated in Hebei Province from 2017 to 2019, of which 1439 strains were CR-ECO (2.65\%). The highest proportion $(33.78 \%)$ of strains was isolated from urine, and the detection rate showed a slow downward trend over the past 3 years. CR-ECO was mainly detected in densely populated and economically developed areas. Of all the patients, $54.2 \%$ were from the medical ward; the ratio of male to female patients with $C R-E C O$ infections was $1.35: 1$; elderly patients and adults accounted for $59.6 \%$ and $30.8 \%$, respectively, whereas minors and newborns accounted for $4.9 \%$ and $4.7 \%$, respectively. For $C R-E C O$, the drug resistance rates to $\beta$ lactams were all higher than $80 \%$ and there was an annual increasing trend, while the drug resistance rates to quinolones remained nearly unchanged. The rate of resistance to aminoglycosides was relatively low, especially to amikacin (approximately 22\%). The MIC50 of other antibacterial drugs, except amikacin, was equal to or higher than the break point of drug resistance.

Conclusion: From 2017 to 2019, the isolation rate of CR-ECO in Hebei Province, China, remained stable; however, the drug resistance rate showed an upward trend, primarily in cases of urinary tract infections in older men; the resistance rate to amikacin was the lowest. Keywords: Enterobacteriales bacteria, multidrug resistance, MIC50/MIC90, regional distribution

\section{Introduction}

Since the advent of penicillin, human beings have discovered and invented various types of antibacterial drugs, which play an extremely important role in the treatment of different infections. However, evidence that reduced susceptibility to antibiotics has been increasing worldwide. ${ }^{1}$ In Spain, the resistance rate of Klebsiella pneumoniae to imipenem increased nearly 13 times from 2010 to $2014 .^{2}$ Data on bacteremia 
patients from 90 Swiss hospitals showed a linear increase in staphylococcus aureus resistance to oxacillin during hospitalization. $^{3}$ From 2010 to 2017, the proportion of Escherichia coli isolated from 25 tertiary hospitals in Greece insensitive to third-generation cephalosporins, carbapenems, and fluoroquinolones was increasing. ${ }^{4}$ There are even strains that are insensitive to many antibiotics, such as "multi-drug-resistant bacteria, pan-drug-resistant bacteria, and super bacteria". 5-8 Carbapenem antibiotics, as broadspectrum $\beta$-lactam drugs, can treat infections caused by Enterobacteriales bacteria and are regarded as the "last line of defense" for infection treatment. ${ }^{9-11}$ Unfortunately, the resistance rate of Enterobacteriales bacteria to carbapenems has been increasing year by year. ${ }^{12-14}$ Carbapenemresistant Enterobacteriales ( $C R E$ ) is a serious public health threat with significant morbidity and mortality, and it has been listed as an urgent threat to bacterial drug resistance by the World Health Organization. ${ }^{15-18}$ In a study of $138 C R E$ infected patients treated with ceftazidime/avibactam, the 30day mortality rate was still as high as $34 \% .{ }^{19} \beta$-lactamase gene transport on mobile components is a key mechanism for the rapid global spread of $C R E .{ }^{11,18}$ With the extensive use of carbapenem antibiotics, carbapenem resistant Escherichia coli (CR-ECO) has evolved, resulting in a dilemma in the selection of antimicrobials for infections with this bacterium. Escherichia coli ST131 producing Klebsiella pneumoniae carbapenemase (KPC) was isolated in Italy. Sequencing showed that the strain contained the kpc-2 variant and TEM-1 $\beta$-lactamase. ${ }^{20}$ In 2020, Escherichia coli ST131 O16: H5 producing oxa-244 was isolated from German patients for the first time. ${ }^{21}$ The spread of such variants thus poses a serious threat to public health. $^{4,21}$ Compared with CRKP, CR-ECO has obviously not attracted enough attention, and its resistance rate, geographical distribution, and other data are unknown in Hebei Province, China. Therefore, the aims of this study were to explore the clinical distribution characteristics and antimicrobial susceptibility of $C R-E C O$ in Hebei Province, China, from 2017 to 2019, and to provide data supporting the treatment of this bacterial infection and the prevention of its spread.

\section{Materials and Methods}

\section{General Information}

The strains were isolated from the urine, sputum, blood, secretions, and other clinical specimens of patients in 43 tertiary hospitals in Hebei Province, China, from 2017 to 2019. A total of $1439 C R-E C O$ strains were obtained. To avoid duplication of statistics in the same patient, the first $C R-E C O$ isolated from this patient was screened with WHONET5.6 software for the study. Escherichia coli ATCC25922 and Pseudomonas aeruginosa ATCC 27853 were obtained from the Clinical Inspection Center of the National Health and Wellness Committee of the People's Republic of China. The bacterial drug sensitivity test recommended by the American Institute of Clinical and Laboratory Standardization (CLSI) was used, and drug sensitivity was determined using the CLSI drug sensitivity break point of the 2019 edition. $^{22}$

\section{Methods}

\section{Instruments and Reagents}

We used the BacT/ALERT 3D120 automatic blood culture instrument (BIO PARTICIPATIONS Co., LTD, Marcyl'étoile, France). The blood culture bottles were obtained from the original factory. The bacterial identification instrument, Phoenix100, FX-200 automatic blood culture instrument (Becton, Dickinson and Company, New Jersey, USA) was used and the biochemical reaction tubes and drug sensitive reaction tubes were all obtained from the original factory. The bacterial solid culture medium used was the MacConkey Agar Medium (Dijing Microbiology Technology Co., LTD, Guangzhou, China).

\section{Research Methods}

The strains were inoculated into the MacConkey Agar Medium at $35{ }^{\circ} \mathrm{C}$ for $24 \mathrm{~h}$. The bacterial solution was prepared to 0.5 McFarland Standards, and $20 \mu \mathrm{L}$ was added to the drug sensitive broth. This was mixed well, and the mixture was poured onto the drug sensitive reaction plate. The remaining bacterial solution was poured onto the biochemical reaction plate. These plates were then placed in the bacterial identification instrument, the Phoenix 100, for the identification and drug sensitivity tests, and the results were read after 24 hours.

The bacterial drug sensitivity test recommended by the American Institute of Clinical and Laboratory Standardization (CLSI) was used, and drug sensitivity was determined using the CLSI drug sensitivity break point of the 2019 edition. CR-ECO was defined as an isolate with imipenem and/or meropenem (MICs $\geq 4 \mu \mathrm{g} / \mathrm{mL}$ ).

\section{Statistical Processing}

WHONET5.6 software was used for data analysis (World Health Organization, Geneva, Switzerland). Images were 
formed with GraphPad prism software (Version 8.4.0, InsightfulScience Co., LTD, San Diego, CA, USA) and Adobe Illustrator 2019 software (Adobe Systems Incorporated Co., LTD, SAN Jose, California, USA).

\section{Results}

A total of 54,377 strains of Escherichia coli were isolated in Hebei Province, China, from 2017 to 2019, including the 1439 strains of CR-ECO (2.65\%). Among the 1439 strains of CR-ECO, 439 strains $(2.81 \%, 439 / 15,599)$ were isolated in 2017, 461 strains $(2.40 \%, 461 / 19,241)$ in 2018 , and 539 strains $(2.76 \%, 539 / 1999)$ in 2019 . Among these, 486 urine samples (33.77\%) ranked first, 419 sputum samples (29.12\%) second, and 131 (9.10\%) blood samples third. Furthermore, 73 catheters and 72 secretions ranked fourth $(5.10 \%)$ and fifth places $(5.00 \%)$, respectively (Table 1).

Between 2017 and 2019, the characteristics of the 1439 patients with CR-ECO infection were as follows: 828 $(57.5 \%)$ males, $611(42.5 \%)$ females, and the ratio of males to females was 1.35:1. There were 858 (59.6\%) older people ( $>60$ years old), $443(30.8 \%)$ adults (18-60 years old), $71(4.9 \%)$ minors (1-18 years old) and 68 (4.7\%) newborns ( $<1$ year old), respectively. The 1439 strains of $C R-E C O$ were isolated from internal medicine 780 (54.20\%), surgery 384 (26.69\%), ICU 139 (9.66\%), pediatrics $80(5.56 \%)$, emergency 43 (2.99\%), and outpatient $11(0.76 \%)$ (Figure 1).

Table I Distribution Characteristics of CR-ECO Specimens from 2017 to 2019

\begin{tabular}{|l|c|c|c|c|c|c|}
\hline \multirow{2}{*}{$\begin{array}{l}\text { Specimen } \\
\text { Type }\end{array}$} & \multicolumn{2}{|c|}{2017} & \multicolumn{2}{c|}{2018} & \multicolumn{2}{c|}{2019} \\
\cline { 2 - 7 } & N & P (\%) & N & P (\%) & N & P (\%) \\
\hline Urine & 152 & 34.6 & 156 & 33.8 & 178 & 33.0 \\
Sputum & 132 & 30.0 & 126 & 27.3 & 161 & 29.8 \\
Blood & 44 & 10.0 & 48 & 10.4 & 39 & 7.2 \\
Catheter & 11 & 2.5 & 26 & 5.6 & 36 & 6.6 \\
Secretion & 15 & 3.4 & 28 & 6.0 & 29 & 5.3 \\
Ascites & 6 & 1.3 & 18 & 3.9 & 21 & 3.8 \\
Pus & 26 & 5.8 & 27 & 5.7 & 16 & 2.9 \\
Bile & 14 & 3.1 & 9 & 1.9 & 8 & 1.4 \\
Drainage & I & 0.2 & 4 & 0.8 & 12 & 2.2 \\
Fecal & 9 & 2.0 & 2 & 0.4 & 3 & 0.5 \\
Vagina & 7 & 1.5 & 0 & 0.0 & 1 & 0.1 \\
Other & 23 & 5.2 & 21 & 4.5 & 35 & 6.4 \\
Total & 439 & 100 & 461 & 100 & 539 & 100 \\
\hline
\end{tabular}

Abbreviations: N, number; P, proportion.
From 2017 to 2019, 1439 strains of $C R-E C O$ were isolated from Hebei Province, China, according to administrative regions, with Shijiazhuang ranking first with 445 strains (30.92\%); Tangshan second with 341 strains (23.70\%); Cangzhou third with 172 strains $(11.95 \%$, and Handan fourth with 103 strains (7.16\%), as shown in Table 2 .

Analysis of trend results of drug resistance rates from 2017 to 2019 yielded the following: all $\beta$-lactamides showed an increasing trend, except ertapenem, which first decreased and then increased. Among the three aminoglycosides, amikacin first increased and then decreased; gentamicin and tobramycin first decreased and then increased; and the two quinolones, levofloxacin and ciprofloxacin, showed an upward trend first and then a downward trend (Table 3).

Analysis of mean drug resistance rates from 2017 to 2019 yielded the following: piperacillin/tazobactam relative to piperacillin (69.5\% vs $92.5 \%)$ and ampicillin/sulbactam relative to ampicillin (89.9\% vs 96.6\%) were significantly reduced; the first to fourth generation cephalosporins represented by cefazolin, cefuroxime, ceftriaxone, cefotaxime, ceftazidime, and cefepime showed a downward trend $(91.3 \%$ vs $91.2 \%$ vs $91.6 \%$ vs $88.2 \%$ vs $85.7 \%$ vs $85.6 \%$ ). Among carbapenems, ertapenem, imipenem, and meropenem were different $(91.3 \%$ vs $77.3 \%$ vs $72.2 \%$ ). Among the three aminoglycosides, gentamicin, tobramycin, and amikacin were significantly different $(65.0 \%$ vs $56.8 \%$ vs $22.2 \%)$. Levofloxacin was slightly lower than ciprofloxacin $(81.0 \%$ vs $84.6 \%)$; compound sulfa was relatively low (76.9\%) (Table 3 ).

\section{Discussion}

In recent years, due to the irregular use of antibacterial drugs, the resistance rate of Escherichia coli to antibacterial drugs has increased. Further, there has been an increase in appearance of multi-drug resistant Escherichia coli. ${ }^{8}$ While carbapenem antibiotics have become the last line of defense against multi-drug resistant Escherichia coli, $C R-E C O$ has appeared all over the world, resulting in difficulties in the treatment of this bacterial infection. $^{13,17,23}$

Carbapenase production is the main mechanism of drug resistance of Enterobacteriales to carbapenems. ${ }^{24}$ Carbapenems can be destroyed by direct hydrolysis. Because most carbapenase genes are located on mobile gene elements, they are easily transferred between Enterobacteriales and other gram-negative bacilli, leading to widespread epidemic spread in a short period of time. ${ }^{25}$ 

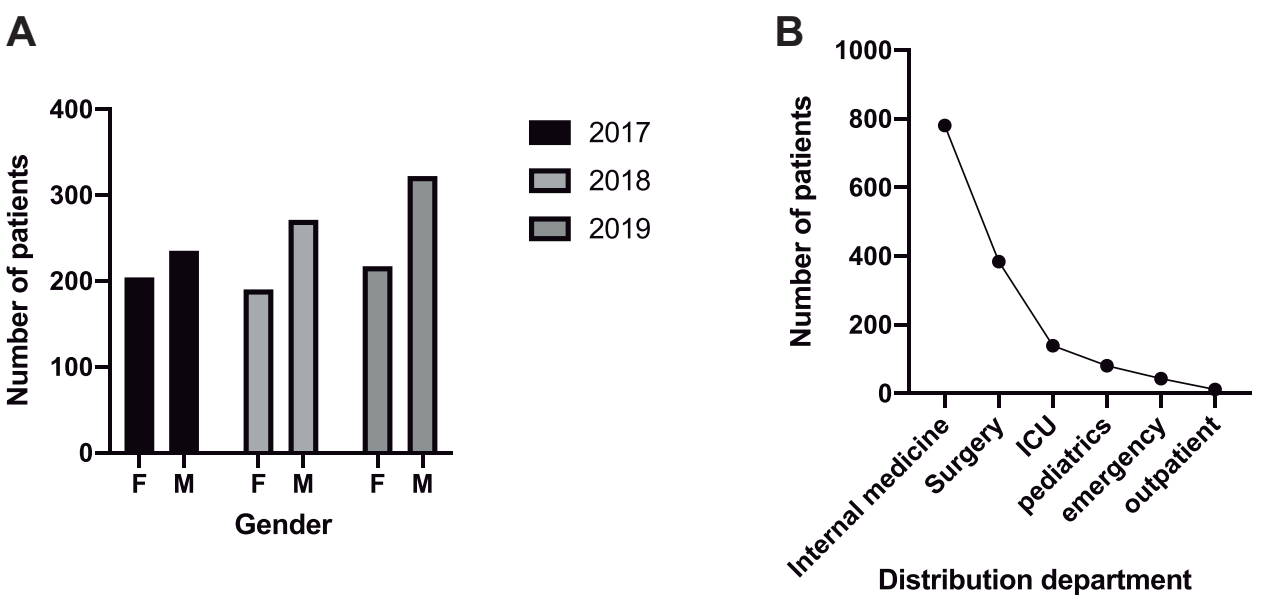

C

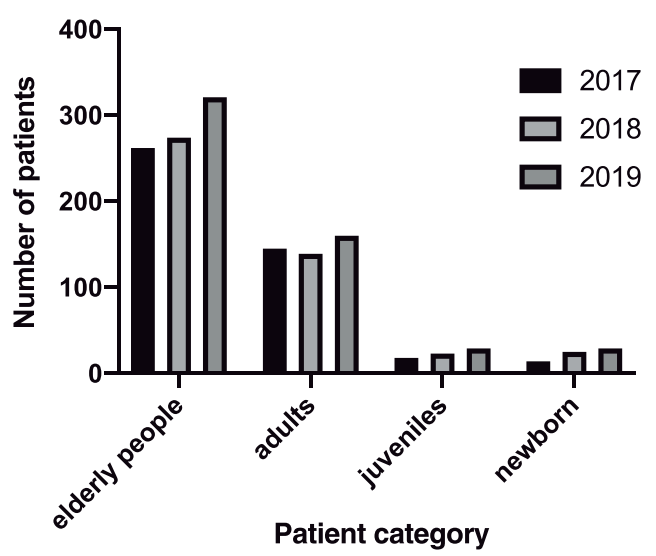

Figure I Clinical distribution characteristics of CR-ECO. (A) Gender distribution characteristics of CR-ECO. (B) Distribution characteristics of CR-ECO departments. (C) Age distribution characteristics of CR-ECO.

Modified carbapenem inactivation method in conjunction with EDTA-modified carbapenem inactivation method and simplified carbapenem Inactivation method can be used to

Table 2 Regional Distribution Characteristics of CR-ECO Specimens from 2017 to 2019

\begin{tabular}{|l|c|c|c|l|c|}
\hline \multirow{2}{*}{ Territory } & \multicolumn{3}{|c|}{ Year } & \multirow{2}{*}{ Total (N) } & Proportion (\%) \\
\cline { 2 - 4 } & $\mathbf{2 0 1 7}$ & $\mathbf{2 0 1 8}$ & $\mathbf{2 0 1 9}$ & & \\
(N) & $\mathbf{( N )}$ & $\mathbf{( N )}$ & & \\
\hline Shijiazhuang & 145 & 142 & 158 & 445 & 30.92 \\
Tangshan & 107 & 113 & 121 & 341 & 23.70 \\
Cangzhou & 29 & 60 & 83 & 172 & 11.95 \\
Handan & 20 & 36 & 47 & 103 & 7.16 \\
Xingtai & 22 & 21 & 18 & 61 & 4.24 \\
Chengde & 28 & 18 & 14 & 60 & 4.17 \\
Qinhuangdao & 19 & 17 & 11 & 47 & 3.27 \\
Zhangjiakou & 7 & 7 & 32 & 46 & 3.20 \\
Langfang & 11 & 11 & 18 & 40 & 2.78 \\
Total & 439 & 461 & 539 & 1439 & 100 \\
\hline
\end{tabular}

Abbreviation: $\mathrm{N}$, number. detect carbapenems. ${ }^{24,26}$ KPC, NDM, IPM, VIM and OXA-48 are common carbapenem resistance genotypes. ${ }^{24,27}$ In 2018, Escherichia coli ST131 producing KPC and TEM carbapenemase was isolated from the urine of patients with urinary tract infection for the first time in Italy. $^{20}$

This study showed that $C R-E C O$ accounted for $2.65 \%$ of all Escherichia coli that caused clinical infections in Hebei Province, China, from 2017 to 2019. From 2009 to 2014, 593 strains of Escherichia coli were isolated from pig feces and rivers in Heilongjiang Province, China, of which $C R$-ECO accounted for $6.74 \% .{ }^{28}$ Moreover, the $C R$ $E C O$ isolated in 10 public pig farms in India accounted for $20.5 \%$ of all the strains of Escherichia coli between 2014 and 2016. ${ }^{29}$ This indicates that $C R-E C O$ spreads in the environment, and that the separation rate is higher than that of human patients. In Hebei Province, China, the highest separation rate of $C R-E C O$ was from urine, accounting for about $33 \%$ of the total. Studies have 
Table 3 Drug Sensitivity Data of CR-ECO to Various Antibacterial Drugs from 2017 to 2019

\begin{tabular}{|c|c|c|c|c|c|c|c|c|c|c|c|c|}
\hline \multirow[t]{2}{*}{ Antibiotic Name } & \multicolumn{3}{|c|}{2017} & \multicolumn{3}{|c|}{2018} & \multicolumn{3}{|c|}{2019} & \multicolumn{3}{|c|}{ Average Value } \\
\hline & $\mathbf{N}$ & R (\%) & S (\%) & $\mathbf{N}$ & R (\%) & S (\%) & $\mathbf{N}$ & $\mathbf{R}(\%)$ & S (\%) & $\mathbf{N}$ & $\mathbf{R}(\%)$ & $\mathbf{S}(\%)$ \\
\hline Ampicillin & 346 & 95.1 & 3.5 & 339 & 97.1 & 1.2 & 471 & 97.2 & 1.7 & 1148 & 96.6 & 2.1 \\
\hline Piperacillin & 278 & 91 & 5.8 & 220 & 91.8 & 3.2 & 226 & 94.7 & 4.4 & 719 & 92.5 & 4.6 \\
\hline Cefazolin & 343 & 84.6 & 15.5 & 383 & 93.5 & 6.5 & 374 & 95.2 & 4.8 & 1092 & 91.3 & 8.7 \\
\hline Cefuroxime & 315 & 88.9 & 9.8 & 343 & 92.1 & 6.4 & 353 & 92.6 & 6.8 & 1004 & 91.2 & 7.7 \\
\hline Ceftriaxone & 322 & 87.6 & 12.1 & 305 & 93.1 & 6.2 & 375 & 93.9 & 5.6 & 996 & 91.6 & 7.9 \\
\hline Cefotaxime & 167 & 81.4 & 18 & 153 & 92.8 & 7.2 & 140 & 91.4 & 8.6 & 458 & 88.2 & 11.6 \\
\hline Compound sulfamethoxazole & 336 & 81 & 19 & 382 & 72.8 & 27.2 & 439 & 77.9 & 22.1 & 1149 & 76.9 & 23.1 \\
\hline Ciprofloxacin & 349 & 80.8 & 16.3 & 408 & 87 & 11.5 & 477 & 85.3 & 10.9 & 1226 & 84.6 & 12.7 \\
\hline Levofloxacin & 349 & 77.7 & 18.1 & 417 & 82.5 & 13.7 & 513 & 82.3 & 14 & $|27|$ & 81.0 & 15.1 \\
\hline Ampicillin/sulbactam & 267 & 92.5 & 4.1 & 368 & 87.5 & 7.1 & 466 & 90.1 & 4.9 & 1093 & 89.8 & 5.5 \\
\hline Gentamicin & 348 & 67 & 30.5 & 414 & 62.1 & 34.3 & 473 & 66.2 & 28.3 & 1227 & 65.0 & 30.9 \\
\hline Tobramycin & 245 & 55.9 & 31.8 & 255 & 53.3 & 29.4 & 248 & 61.7 & 19.4 & 743 & 56.8 & 27.1 \\
\hline Aztreonam & 283 & 82.3 & 15.2 & 236 & 77.5 & 19.9 & 286 & 82.5 & 16.4 & 801 & 81.0 & 17 \\
\hline Cefepime & 352 & 84.9 & 13.4 & 426 & 84.5 & 10.6 & 514 & 87.2 & 8 & 1284 & 85.6 & 10.4 \\
\hline Ceftazidime & 351 & 79.5 & 18.5 & 344 & 89 & 9.6 & 510 & 88 & 10 & 1197 & 85.7 & 12.4 \\
\hline Amoxicillin/clavulanic acid & 137 & 39.4 & 49.6 & 97 & 89.7 & 8.2 & 120 & 86.7 & 9.2 & 353 & 69.1 & 24.6 \\
\hline Piperacillin/tazobactam & 355 & 62.5 & 31.3 & 422 & 69 & 18.5 & 507 & 75.1 & 16.2 & 1276 & 69.5 & 21.2 \\
\hline Meropenem & 361 & 62.3 & 32.7 & 402 & 74.9 & 17.9 & 472 & 78 & 18.2 & 1227 & 72.2 & 22.4 \\
\hline Imipenem & 357 & 66.1 & 30.5 & 424 & 79.7 & 13 & 511 & 82.8 & 12.7 & 1284 & 77.3 & 17.8 \\
\hline Ertapenem & 91 & 91.2 & 8.8 & 88 & 90.9 & 9.1 & 84 & 91.7 & 7.1 & 263 & 91.3 & 8.3 \\
\hline Amikacin & 351 & 22.8 & 72.9 & 425 & 35.5 & 58.6 & 513 & 19.3 & 78.6 & $|28|$ & 22.2 & 70.6 \\
\hline
\end{tabular}

Abbreviations: $\mathrm{N}$, number of strains; $\mathrm{R}$, drug resistance rate; $\mathrm{S}$, sensitivity rate.

shown that the most common pathogen found in urine is Escherichia coli, of which $70.3 \%$ are multi-drug resistant bacteria, $1.65 \%$ CR-ECO, while $0.8 \%$ produce the blaOXA-48 gene. ${ }^{30}$ In Hebei Province, China, there are more male than female patients with $C R-E C O$ infection (1.35 vs 1), while in the United States, $59 \%$ were women. ${ }^{31}$ In addition, the majority (90.4\%) of the $C R$ ECO infected people in Hebei Province, China, are older persons and adults, with only $9.6 \%$ being newborns and minors. According to the statistics of two teaching hospitals in London, $51.2 \%$ of the CR-ECO patients are between 16 and 64 years old, while $48.8 \%$ patients were over 64 years old. ${ }^{12}$ The median age of the CR-ECO infected people in the United States was 66 years old. ${ }^{31}$ According to the above data, $C R-E C O$ causes infections mainly in older patients. In this study, the CR-ECO inpatients accounted for $99.2 \%$, with the highest proportion being in internal medicine, exceeding $50 \%$, while the ICU accounted for approximately $10 \%$. Johnson et $\mathrm{al}^{32}$ showed that patients with $C R-E C O$ infection mainly were admitted to the ICU. In Table 2, the CR-ECO isolated clinically from Shijiazhuang and Tangshan in 2017-2019 showed first and second places in all the cities in Hebei Province, China, respectively, while the total proportions of the two cities exceeded half of the province; Langfang and Zhangjiakou were the last and penultimate places, respectively. The total proportion was about $6 \%$. In 2019, the China Antimicrobial Resistance Surveillance Network (CARSS) showed that the top five provinces and cities in China with respect to the $C R-E C O$ separation rates were Beijing, Liaoning, Henan, Jiangsu, and Shanghai, and the five provinces and cities with the lowest separation rates were Tibet, Gansu, Ningxia, Inner Mongolia, and Shanxi. In America, the $C R-E C O$ separation rates in Georgia, Maryland, and New York were significantly higher than those in Colorado, New Mexico, and Oregon. ${ }^{31}$ This indicates that $C R-E C O$ is distributed mainly in densely populated and economically developed areas.

At present, the antibiotics used to treat $C R E$ are limited. Polymyxin, tigecycline, fosfomycin and aminoglycosides are the drugs of choice for the treatment of CRE infections; however their clinical use is limited by their pharmacokinetics and side effects. ${ }^{13,17,33}$ The drug resistance rate of piperacillin/tazobactam was $23 \%$ lower 
than that of piperacillin, and the drug resistance rate of ampicillin/sulbactam was $6.8 \%$ lower than that of ampicillin, which proved that the antibacterial activity of penicillin plus enzyme inhibitor was higher than that of a single drug preparation. For carbapenem-sensitive Escherichia coli, the resistance rate of piperacillin/tazobactam is less than one tenth of that of piperacillin. ${ }^{34}$ In this study, the resistance rate of the first to the fourth generation cephalosporins to $C R-E C O$ showed a downward trend, indicating that the level of cephalosporins increased with the increase in antibacterial activity. However, it is worth noting that the overall drug resistance rate of $C R-E C O$ to cephalosporins is still very high, over $85 \%$. Among the third generation cephalosporins, the antibacterial activity of ceftazidime against $C R-E C O$ was superior to that of cefotaxime and ceftriaxone. In North America, the sensitivity of ceftazidime/avibactam to $C R-E C O$ was much higher than that of ceftazidime $(92 \%$ vs $19 \%){ }^{35}$ In this study, the drug resistance rates of $C R-E C O$ to ertapenem, imipenem, and meropenem were $91.3 \%, 77.3 \%$, and $72.2 \%$, respectively. Johnston et $\mathrm{al}^{35}$ showed that the sensitivity rates of meropenem, imipenem, and ertapenem were $59 \%$, $29 \%$, and $4 \%$, respectively. It can be seen that the antibacterial activity of the above three carbapenems against CR-ECO descending order is meropenem, imipenem, and ertapenem. When treating infections caused by multidrug-resistant gram-negative bacteria, aminoglycosides are recommended, especially for urinary tract infections caused by renal accumulation. Considering the ototoxicity and nephrotoxicity of such drugs, drug MIC should be monitored for individualized treatment. ${ }^{13}$ In this study, the antibacterial activity of amikacin against $C R-E C O$ was 41.1 and 42.4 percentage points higher than that of tobramycin and gentamicin, respectively. Amikacin showed higher antimicrobial activity against CR-ECO than gentamicin in Asia-Pacific $(68 \%$ vs $31 \%$ ) and Europe (78\% vs 50\%). ${ }^{35}$ The resistance rates of $C R-E C O$ to gentamicin and amikacin in China were $59.3 \%$ and $13.2 \%$, respectively. ${ }^{33}$ Amikacin has shown high activity against $C R-E C O$ and can be used as an empirical drug candidate for the treatment of $C R$ $E C O$ infections. However, we should pay close attention to its adverse reactions such as ototoxicity and nephrotoxicity.

The MIC50/MIC90 refers to the minimum inhibitory concentration of antibacterial drugs that can inhibit the growth of pathogens by $50 \% / 90 \% .^{36}$ In this study
(Table 4), the CR-ECO MIC50 in Hebei Province, China, was equal to or slightly higher than the resistance break points of most antibiotics. The MIC50 of cefotaxime and ceftriaxone was 16 times that of the resistance break point, but the MIC50 of amikacin was far lower than that of the resistance break point, which was $6.25 \%$ of the resistance break point and $25 \%$ of the sensitivity break point. In this study, amikacin had a higher activity against $C R-E C O$ than other antibacterial drugs (Table 3). Unfortunately, $C R-E C O$ still showed a high drug resistance rate to new antibacterial drugs, with sensitivity rates of $45 \%$ (MIC50 $\geq 256 \mu \mathrm{g} / \mathrm{mL}$ ) and only $10 \%$ (MIC50 $\geq 256 \mu \mathrm{g} / \mathrm{mL}$ ) to ceftazidime/ tazobactam. ${ }^{37}$

\section{Conclusion}

In conclusion, the detection rate of $C R-E C O$ in Hebei Province, China, remained relatively stable from 2017 to 2019, while the drug resistance to commonly used antibiotics in the clinic was high, especially the drug resistance rate to penicillins and cephalosporins, which was as high as $90.0 \%$. To treat this bacterial infection, sensitive antibacterial agents should be selected according to the results of drug sensitivity tests. $C R$ $E C O$ mainly causes urinary tract infections in older male inpatients. In addition, $C R-E C O$ is distributed mainly in densely populated and economically developed areas in Hebei Province, China. To understand the epidemiological characteristics of the $C R-E C O$ area, it is necessary to monitor drug resistance data. A limitation of this study is that the isolates were not sequenced for drug-resistant genotypes, so the transmission trajectories could not be accurately tracked. The administration of carbapenems should be strengthened to delay the generation of $C R-E C O$. To prevent the spread of $C R-E C O$, medical and government agencies need to work together to develop scientific prevention and control measures.

\section{Data Sharing Statement}

The data used and/or analyzed in this study are available from the corresponding author on reasonable request.

\section{Ethics Approval and Informed Consent}

The protocol has been reviewed by the Ethics Committee (IRB) of the First Affiliated Hospital of Hebei North University. Since the project is an observational study and all 
Table 4 CR-ECO MIC50 and MIC90 in 2017-2019

\begin{tabular}{|c|c|c|c|c|c|}
\hline Antibiotic Name & Quantity & Break Point & MIC50 & MIC90 & MIC Range \\
\hline Tigecycline & 159 & $S \leq 0.03 R \geq 0.25$ & 0.5 & 1 & $0.025-8$ \\
\hline Ertapenem & 262 & $S \leq 0.5 R \geq 2$ & 2 & 8 & $0.12-32$ \\
\hline Cefotaxime & 458 & $S \leq I R \geq 4$ & 64 & 64 & $0.12-128$ \\
\hline Piperacillin & 719 & $S \leq 16 R \geq 128$ & 128 & 128 & $2-256$ \\
\hline Tobramycin & 743 & $S \leq 4 R \geq 16$ & 16 & 16 & $0.5-32$ \\
\hline Aztreonam & 801 & $S \leq 4 R \geq 16$ & 32 & 64 & $0.25-128$ \\
\hline Ceftriaxone & 996 & $S \leq I R \geq 4$ & 64 & 128 & $0.25-128$ \\
\hline Cefazolin & 1092 & $S \leq 16 R \geq 32$ & 32 & 64 & $2-128$ \\
\hline Ampicillin/sulbactam & 1093 & $S \leq 8 R \geq 32$ & 32 & 64 & $2-128$ \\
\hline Ampicillin & 1148 & $S \leq 8 R \geq 32$ & 32 & 64 & $2-128$ \\
\hline Compound sulfamethoxazole & 1149 & $S \leq 2 R \geq 4$ & 8 & 16 & $0.25-32$ \\
\hline Ceftazidime & 1197 & $S \leq 4 R \geq 16$ & 32 & 64 & $0.12-128$ \\
\hline Ciprofloxacin & 1226 & $S \leq I R \geq 4$ & 4 & 4 & $0.06-32$ \\
\hline Meropenem & 1227 & $S \leq I R \geq 4$ & 8 & 16 & $0.06-32$ \\
\hline Gentamicin & 1227 & $S \leq 4 R \geq 16$ & 16 & 16 & $0.5-32$ \\
\hline Levofloxacin & $127 \mid$ & $S \leq 2 R \geq 8$ & 8 & 16 & $0.12-16$ \\
\hline Piperacillin/tazobactam & 1276 & $S \leq 16 R \geq 128$ & 128 & 128 & $\mathrm{I}-256$ \\
\hline Amikacin & $|28|$ & $S \leq 16 R \geq 64$ & 4 & 64 & $0.25-128$ \\
\hline Cefepime & 1284 & $S \leq 2 R \geq 16$ & 32 & 64 & $0.12-128$ \\
\hline Imipenem & 1284 & $S \leq I R \geq 4$ & 8 & 16 & $0.0625-64$ \\
\hline
\end{tabular}

Abbreviations: MIC, minimum inhibitory concentration; MIC50/MIC 90 , MIC50 and MIC 90 can inhibit $50 \%$ or $90 \%$ of the tested bacteria.

bacterial strains are from strains cultured from residual samples used in clinical diagnosis, it involves the confidentiality of patient data and compliance with the Declaration of Helsinki. Since the data did not affect patient care, it was determined that the exemption criteria were met. After consulting the IRB of the First Affiliated Hospital of Hebei North University, a formal ethical review was approved, and written informed consent from the patient was not required (ethical approval No.: K2019147).

\section{Acknowledgments}

The author thanks all his colleagues who have helped this project, and thanks Editage for English language editing.

\section{Author Contributions}

All authors contributed to data analysis, drafting or revising article agree to submit the articles to infection and drug resistance magazine, finally approve the version to be published, and agree to be accountable for all aspects of the work.

\section{Funding}

This work was supported by the Youth Science and Technology Project from Department of Health of Hebei Province in China (registration number: 20180843, 20190904 and 20210702) and Key R\&D project of Zhangjiakou City (2121098D, 2121064D).

\section{Disclosure}

The authors report no conflicts of interest in this work.

\section{References}

1. Acevedo R, Bai X, Borrow R, et al. The global meningococcal initiative meeting on prevention of meningococcal disease worldwide: epidemiology, surveillance, hypervirulent strains, antibiotic resistance and high-risk populations. Expert Rev Vaccines. 2019;18(1):15-30. doi:10.1080/14760584.2019.1557520

2. Aracil-García B, Oteo-Iglesias J, Cuevas-Lobato Ó, et al. Rapid increase in resistance to third generation cephalosporins, imipenem and co-resistance in Klebsiella pneumoniae from isolated from 7140 blood-cultures (2010-2014) using EARS-Net data in Spain. Enferm Infecc Microbiol Clin. 2017;35(8):480-486. doi:10.1016/j. eimc.2016.06.007

3. Buetti N, Marschall J, Timsit JF, Atkinson A, Kronenberg A, Sommerstein R. Distribution of pathogens and antimicrobial resistance in bacteraemia according to hospitalization duration: a nationwide surveillance study in Switzerland. Clin Microbiol Infect. 2021. doi:10.1016/j.cmi.2021.04.025

4. Polemis M, Tryfinopoulou K, Giakkoupi P, Vatopoulos A. Eight-year trends in the relative isolation frequency and antimicrobial susceptibility among bloodstream isolates from Greek hospitals: data from the Greek electronic system for the surveillance of antimicrobial resistance - WHONET-Greece, 2010 to 2017. Euro Surveill. 2020;25(34). doi:10.2807/1560-7917.ES.2020.25.34.1900516

5. Liu Y, Li R, Xiao X, Wang Z. Antibiotic adjuvants: an alternative approach to overcome multi-drug resistant Gram-negative bacteria. Crit Rev Microbiol. 2019;45(3):301-314. doi:10.1080/1040841X.2019.1599813

6. Mühlberg E, Umstätter F, Kleist C, Domhan C, Mier W, Uhl P. Renaissance of vancomycin: approaches for breaking antibiotic resistance in multidrug-resistant bacteria. Can J Microbiol. 2020;66 (1):11-16. doi:10.1139/cjm-2019-0309 
7. Zhang J, Xu J, Ma H, et al. Designing an amino-fullerene derivative C(70)-(EDA)(8) to fight superbacteria. ACS Appl Mater Interfaces. 2019;11(16):14597-14607. doi:10.1021/acsami.9b01483

8. Wu B, Qi C, Wang L, et al. Detection of microbial aerosols in hospital wards and molecular identification and dissemination of drug resistance of Escherichia coli. Environ Int. 2020;137:105479. doi:10.1016/j.envint.2020.105479

9. Nordmann P, Poirel L. Epidemiology and diagnostics of carbapenem resistance in gram-negative bacteria. Clin Infect Dis. 2019;69(Suppl 7):S521-s528. doi:10.1093/cid/ciz824

10. Doi Y. Treatment options for carbapenem-resistant gram-negative bacterial infections. Clin Infect Dis. 2019;69(Suppl 7):S565-s575. doi:10.1093/cid/ciz830

11. Potter RF, D'Souza AW, Dantas G. The rapid spread of carbapenem-resistant Enterobacteriaceae. Drug Resist Updat. 2016;29:30-46. doi:10.1016/j.drup.2016.09.002

12. Freeman R, Moore LS, Charlett A, Donaldson H, Holmes AH. Exploring the epidemiology of carbapenem-resistant Gram-negative bacteria in west London and the utility of routinely collected hospital microbiology data. J Antimicrob Chemother. 2015;70(4):1212-1218. doi:10.1093/jac/dku500

13. Fritzenwanker M, Imirzalioglu $C$, Herold S, Wagenlehner FM, Zimmer KP, Chakraborty T. Treatment options for carbapenemresistant gram-negative infections. Dtsch Arztebl Int. 2018;115(2021):345-352. doi: 10.3238/arztebl.2018.0345

14. Wang Q, Wang X, Wang J, et al. Phenotypic and genotypic characterization of carbapenem-resistant Enterobacteriaceae: data from a longitudinal large-scale CRE Study in China (2012-2016). Clin Infect Dis. 2018;67(suppl_2):S196-s205. doi:10.1093/cid/ciy660

15. Cassini A, Högberg LD, Plachouras D, et al. Attributable deaths and disability-adjusted life-years caused by infections with antibiotic-resistant bacteria in the EU and the European Economic Area in 2015: a population-level modelling analysis. Lancet Infect Dis. 2019;19(1):56-66. doi:10.1016/S1473-3099(18)30605-4

16. Peri AM, Doi Y, Potoski BA, Harris PNA, Paterson DL, Righi E. Antimicrobial treatment challenges in the era of carbapenem resistance. Diagn Microbiol Infect Dis. 2019;94(4):413-425. doi:10.1016/j.diagmicrobio.2019.01.020

17. Wenzler E, Scoble PJ. An appraisal of the pharmacokinetic and pharmacodynamic properties of meropenem-vaborbactam. Infect Dis Ther. 2020;9(4):769-784. doi:10.1007/s40121-020-00344-Z

18. Logan LK, Weinstein RA. The epidemiology of carbapenem-resistant Enterobacteriaceae: the impact and evolution of a global menace. $J$ Infect Dis. 2017;215(suppl_1):S28-s36. doi:10.1093/infdis/jiw282

19. Tumbarello M, Trecarichi EM, Corona A, et al. Efficacy of Ceftazidime-Avibactam salvage therapy in patients with infections caused by Klebsiella pneumoniae Carbapenemase-producing K. pneumoniae. Clin Infect Dis. 2019;68(3):355-364. doi:10.1093/cid/ciy492

20. Ripabelli G, Sammarco ML, Scutellà M, Felice V, Tamburro M. Carbapenem-resistant KPC- and TEM-producing Escherichia coli ST131 isolated from a hospitalized patient with urinary tract infection: first isolation in Molise Region, Central Italy, July 2018. Microb Drug Resist. 2020;26(1):38-45. doi:10.1089/mdr.2019.0085

21. Welker S, Boutin S, Miethke T, Heeg K, Nurjadi D. Emergence of carbapenem-resistant ST131 Escherichia coli carrying bla (OXA-244) in Germany, 2019 to 2020. Euro Surveill. 2020;25(46). doi:10.2807/1560-7917.ES.2020.25.46.2001815

22. Wayne P. The Clinical and Laboratory Standards Institute antimicrobial susceptibility testing standards. CLSI. 2019;3:M100-ED29.
23. Diallo OO, Baron SA, Abat C, Colson P, Chaudet H, Rolain JM. Antibiotic resistance surveillance systems: a review. $J$ Glob Antimicrob Resist. 2020;23:430-438. doi:10.1016/j.jgar.2020.10.009

24. Patil S, Chen H, Zhang X, Lian M, Ren PG, Wen F. Antimicrobial resistance and resistance determinant insights into multi-drug resistant gram-negative bacteria isolates from paediatric patients in China. Infect Drug Resist. 2019;12:3625-3634. doi:10.2147/IDR.S223736

25. Spaziante M, Venditti C, Butera O, et al. Importance of surveillance of new delhi metallo-beta-lactamase Klebsiella pneumoniae: molecular characterization and clonality of strains isolated in the Lazio Region, Italy. Infect Drug Resist. 2021;14:3659-3665. doi:10.2147/IDR.S318717

26. Wang YLH, Zhang L, Sun B. Application of modified carbapenem inactivation method and its derivative tests for the detection of carbapenemase-producing Aeromonas. Infect Drug Resist. 2021;2021(14):3949-3960. doi: 10.2147/IDR.S330115

27. El-Badawy MF, Abdelwahab SF, Alghamdi SA, Shohayeb MM. Characterization of phenotypic and genotypic traits of carbapenem-resistant Acinetobacter baumannii clinical isolates recovered from a tertiary care hospital in Taif, Saudi Arabia. Infect Drug Resist. 2019;12:3113-3124. doi:10.2147/IDR.S206691

28. Cheng P, Li F, Liu R, et al. Prevalence and molecular epidemiology characteristics of carbapenem-resistant Escherichia coli in Heilongjiang Province, China. Infect Drug Resist. 2019;12:2505-2518. doi:10.2147/ IDR.S208122

29. Pruthvishree BS, Vinodh Kumar OR, Sinha DK, et al. Spatial molecular epidemiology of carbapenem-resistant and New Delhi metallo beta-lactamase (blaNDM)-producing Escherichia coli in the piglets of organized farms in India. J Appl Microbiol. 2017;122(6):1537-1546. doi:10.1111/jam.13455

30. Gurung S, Kafle S, Dhungel B, et al. Detection of OXA-48 gene in carbapenem-resistant Escherichia coli and Klebsiella pneumoniae from urine samples. Infect Drug Resist. 2020;13:2311-2321. doi:10.2147/IDR.S259967

31. Guh AY, Bulens SN, Mu Y, et al. Epidemiology of carbapenemresistant Enterobacteriaceae in 7 US communities, 2012-2013. JAMA. 2015;314(14):1479-1487. doi:10.1001/jama.2015.12480

32. Johnson JK, Robinson GL, Pineles LL, et al. Carbapenem MICs in Escherichia coli and Klebsiella species producing extended-spectrum $\beta$-lactamases in critical care patients from 2001 to 2009. Antimicrob Agents Chemother. 2017;61(4). doi:10.1128/AAC.01718-16

33. Tang X, Xiao M, Zhuo C, Xu Y, Zhong N. Multi-level analysis of bacteria isolated from inpatients in respiratory departments in China. J Thorac Dis. 2018;10(5):2666-2675. doi:10.21037/jtd.2018.04.46

34. Hu FP, Guo Y, Zhu DM, et al. Resistance trends among clinical isolates in China reported from CHINET surveillance of bacterial resistance, 2005-2014. Clin Microbiol Infect. 2016;22(Suppl 1):S914. doi:10.1016/j.cmi.2016.01.001

35. Johnston BD, Thuras P, Porter SB, et al. Activity of cefiderocol, ceftazidime-avibactam, and eravacycline against carbapenem-resistant Escherichia coli isolates from the United States and international sites in relation to clonal background, resistance genes, coresistance, and region. Antimicrob Agents Chemother. 2020;64(10):451. doi:10.1128/ AAC.00797-20

36. Garcia-Effron G. Rezafungin-mechanisms of action, susceptibility and resistance: similarities and differences with the other echinocandins. J Fungi (Basel). 2020;6(4):67. doi: 10.3390/jof6040262

37. Alatoom A, Elsayed H, Lawlor K, et al. Comparison of antimicrobial activity between ceftolozane-tazobactam and ceftazidime-avibactam against multidrug-resistant isolates of Escherichia coli, Klebsiella pneumoniae, and Pseudomonas aeruginosa. Int $J$ Infect Dis. 2017;62:39-43. doi:10.1016/j.ijid.2017.06.007 


\section{Publish your work in this journal}

Infection and Drug Resistance is an international, peer-reviewed openaccess journal that focuses on the optimal treatment of infection (bacterial, fungal and viral) and the development and institution of preventive strategies to minimize the development and spread of resistance. The journal is specifically concerned with the epidemiology of antibiotic resistance and the mechanisms of resistance development and diffusion in both hospitals and the community. The manuscript management system is completely online and includes a very quick and fair peerreview system, which is all easy to use. Visit http://www.dovepress.com/ testimonials.php to read real quotes from published authors.

Submit your manuscript here: https://www.dovepress.com/infection-and-drug-resistance-journal 